\title{
Preliminary Study on the Preventive Effect of Ginsenoside Rg1 on Posttraumatic Stress Disorder in Mice
}

\author{
Yingchang Lu $^{1}$
}

\begin{abstract}
${ }^{1}$ Department of Physiology and Pathophysiology, School of Basic Medical Sciences, Qingdao University, Qingdao, Shandong, 266071, China

Abstract: Objective To study the preventive effect of ginsenoside Rg1 on posttraumatic stress disorder (PTSD) in mice. Methods Single prolonged stress (SPS) animal model was adopted. Mice treated with normal saline, ginsenoside $(\operatorname{Rg} 1)$ and sertraline after stress were divided into negative control group(SPS+NS), experimental group(SPS+Rg1) and positive control group(SPS+Sertraline) respectively, and a blank control group(NS) was set. On the 8th and 10th days after modeling, the viaduct cross maze experiment and the forced swimming experiment were respectively carried out. Results In the elevated plus maze experiment, the percentage of opening times of mice in the experimental group and the negative control group $[(21.11 \pm 7.21) \%,(20.80 \pm 9.06) \%]$ was significantly lower than that in the blank control group and the positive control group $[(44.99 \pm 7.69) \%,(39.35 \pm 14.14) \%, P<0.0001]$.In the forced swimming experiment, the immobility time of mice in the experimental group and the negative control group $[(64.05 \pm 24.27) \mathrm{s},(51.23 \pm 19.76) \mathrm{s}]$ was significantly higher than that in the blank control group and the positive control group [(16.85 \pm 6.90$) \mathrm{s},(33.26 \pm 20.30) \mathrm{s}, \mathrm{P}<0.001]$. Conclusion The normal dose of ginsenoside $\mathrm{Rg} 1$ could not alleviate the anxiety and fear responses in the mice with post-traumatic stress disorder.
\end{abstract}

Keywords: Ginsenoside, Post-Traumatic Stress Disorder, Single Prolonged Stress

\section{Introduction}

Post-traumatic stress disorder (PTSD) is a delayed and long-lasting mental disorder caused by life threatening events or severe trauma. Its symptoms mainly include pathological recurrence of traumatic events, avoidance of trauma-related clues, persistent high arousal, and emotional numbness ${ }^{1}$. PTSD has multiple causes and serious harms. Selective serotonin reuptake inhibitor (SSRIs) is currently recognized as the first-line treatment for acute and chronic PTSD $^{2}$.However, long-term use of SSRIs has some side effects, including abnormal reactions of the central nervous system, gastrointestinal dysfunction and sexual dysfunction ${ }^{3}$.Previous studies have shown that ginsenoside $\mathrm{Rg} 1$, as an important active component of ginseng, belongs to the original ginseng triol type (see figure 1), has no toxic and side effects, and has neuroprotective and neurotrophic effects ${ }^{4,5}$, which can promote neurogenesis ${ }^{6}$. The purpose of this study was to investigate the preventive effect of ginsenoside $\mathrm{Rg} 1$ on PTSD in mice.

\section{Materials and Methods \\ The Material}

1. The animals: healthy male C57BL6 mice south model biology research center (Shanghai), 10-12 weeks, weight of $25 \sim 30 \mathrm{~g}$, came here, 32, only $12 \mathrm{~h}, 12$ $\mathrm{h} /$ light/dark cycle $22 \sim 25{ }^{\circ} \mathrm{C}$, environment temperature humidity between $65 \sim 75 \%$, free to eat drink, to adapt to at least 1 week after the experiment.

2. Drugs and reagents: ginseng saponin $\operatorname{Rg} 1$ (chemical research center to provide basic medical college of Jilin university, purity > 98\%), Sertraline (Tocris products), bromine DNA nucleoside uracil (Bromodeoxyuridine, Brdu) (Sigma products), rat anti Brdu monoclonal antibody (Accurate) Chemicals products, chicken to sustain mouse IgG (Invitrogen products).

\section{The method}

1. Model establishment: Single prolonged stress (SPS) modeling method developed by Liberzon et al was adopted $^{7}$. The mice were fixed for $2 \mathrm{~h}$.After $20 \mathrm{~min}$ immediately forced swimming, water temperature $24{ }^{\circ} \mathrm{C}$.Rest for 15 minutes after the use of ether anesthesia to complete loss of consciousness; Finally the mice were put back into the cage.

2. Grouping and administration: mice were randomly divided into four groups, 8 mice in each group.(1) blank control group (sham, NS) : intraperitoneal injection of normal saline $(10 \mathrm{mg} / \mathrm{kg} / \mathrm{d})$, once a day for 8d;(2) negative control group (SPS+NS after modeling) : intraperitoneal injection of normal saline $(10 \mathrm{mg} / \mathrm{kg} / \mathrm{d})$, once a day for $8 \mathrm{~d} ;(3)$ experimental group (injection of ginsenoside $\operatorname{Rg} 1$, SPS+Rg1 after modeling) : intraperitoneal injection of ginsenoside

This article is published under the terms of the Creative Commons Attribution License 4.0 Author(s) retain the copyright of this article. Publication rights with Alkhaer Publications.

Published at: http://www.ijsciences.com/pub/issue/2019-05/

DOI: 10.18483/ijSci.2088; Online ISSN: 2305-3925; Print ISSN: 2410-4477 
$(10 \mathrm{mg} / \mathrm{kg} / \mathrm{d}$, normal saline solution), once a day for 8d;(4) positive control group (SPS+Sertraline after modeling) : Sertraline was intraperitoneally injected $(10 \mathrm{mg} / \mathrm{kg} / \mathrm{d}$, normal saline solution) once a day for $8 \mathrm{~d}$.

3. Behavioral measurement: (1) The elevated plus maze experiment(EPM): cameras were placed above the laboratory to monitor the activity of animals in the behavioral test, and video was collected for software analysis and the behavioral indicators were derived. The experiment was carried out on the eighth day after modeling. Referring to literature ${ }^{8}$ and slightly modified, the mice were placed in the center with the head towards the open arm, and their movements were recorded for 10min.Behavioral indicators such as residence time in the open arm and times of entering the open arm were recorded.(2) Forced swimming experiment(FS): referring to literature ${ }^{9}$, the experiment was carried out on the 10th day after modeling. Experiment container assumes the circular, $20 \mathrm{~cm}, 12$ $\mathrm{cm}$ in diameter, depth of $10 \mathrm{~cm}$, water temperature $24{ }^{\circ} \mathrm{C}$, observe the $10 \mathrm{~min}$, record the motionless time of mice and first time incubation period of the state. The standard for immobility is that the mice float, with only small body movements to maintain balance.

4. Statistical analysis: Data were expressed as mean \pm S.E.M. ANOVAs were used for statistical comparisons between groups as described in the main text. The significance level was set to $\mathrm{P}<0.05$.

\section{Results}

1. Comparison of experimental results of elevated plus maze(EPM) in mice in each group

There were significant differences in the percentage of time of entry (Fig.1A;Ordinary one-way ANOVA analysis, $\mathrm{F}_{(3,28)}=12.64, \mathrm{P}<0.0001$.) and residence of the four groups (Fig.1B;Ordinary one-way ANOVA analysis, $\left.\mathrm{F}_{(3,28)}=5.752, \mathrm{P}<0.01\right)$. There was no significant difference between the SPS+NS group and the SPS+Rg1 group (Fig.1A; Tukey's multiple

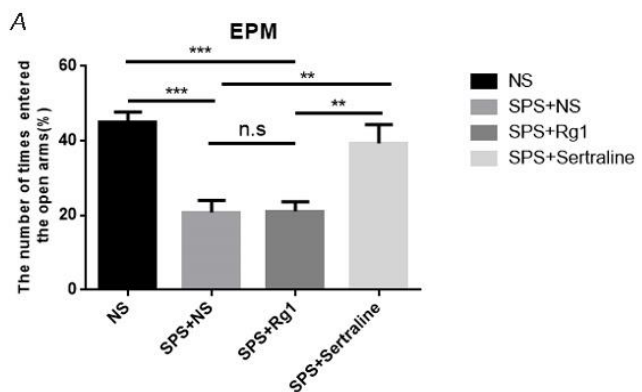

comparisons test, $\mathrm{P}>0.05, \mathrm{n}=8$ ). The percentages of open arm entry times in the SPS+NS group and the SPS $+\mathrm{Rg} 1$ group were significantly reduced compared with the NS and SPS+Sertraline group(Fig.1A; Tukey's multiple comparisons test, $\mathrm{P}<0.001$ for NS vs. SPS+NS, $\mathrm{P}<0.001$ for NS vs. SPS+Rg1, $\mathrm{P}<0.001$ for $\mathrm{SPS}+\mathrm{NS}$ vs. SPS+Sertraline, $\mathrm{P}<0.01$ for $\mathrm{SPS}+\mathrm{Rg} 1$ vs. SPS+Sertraline, $n=8$ ). The percentage of open arm residence time in the SPS+NS group and the SPS+Rg1 group were significantly reduced compared with the NS group(Fig.1B; Tukey's multiple comparisons test, $\mathrm{P}<0.05$ for NS vs. SPS+NS, $\mathrm{P}<0.05$ for NS vs. SPS $+\operatorname{Rg} 1, n=8)$.. Compared with the SPS+Sertraline group, the percentage of open arm residence time in the SPS+Rg1 group was significantly reduced (Fig.1B; Tukey's multiple comparisons test, $\mathrm{P}<0.05$ for SPS+Rg1 vs. SPS+Sertraline, $n=8 P$ ).

\section{Comparison of the results of forced swimming(FS) among the groups}

4 groups of mice showed significant differences in immobility time (Fig.2A;Ordinary one-way ANOVA analysis, $\mathrm{F}_{(3,28)}=9.469, \mathrm{P}<0.001$.) and incubation period (Fig.2B; Ordinary one-way ANOVA analysis, F $(3,28)=3.704, \mathrm{P}<0.05$.). There was no significant difference between the SPS+NS group and the SPS+Rg1 group (Fig.2A and B; Tukey's multiple comparisons test, $\mathrm{P}>0.05, \mathrm{n}=8)$. Compared with NS group, the immobility time of SPS+NS group and SPS+Rg1 group was significantly prolonged (Fig.2A; Tukey's multiple comparisons test, $\mathrm{P}<0.01$ for NS vs. SPS + NS, $\quad \mathrm{P}<0.001$ for NS vs. SPS+Rg1, $n=8)$.Compared with the SPS+Sertraline group, the mice in the SPS+Rg1 group also had significantly longer inactivity time (Fig.1A; Tukey's multiple comparisons test, $\mathrm{P}<0.05$ for SPS+Rg1 vs. SPS+Sertraline, $n=8$ ). Compared with the NS group, the latency time of the SPS+Rg1 group was significantly reduced (Fig.1B; Tukey's multiple comparisons test, $\mathrm{P}<0.05, \mathrm{n}=8 \mathrm{P})$.

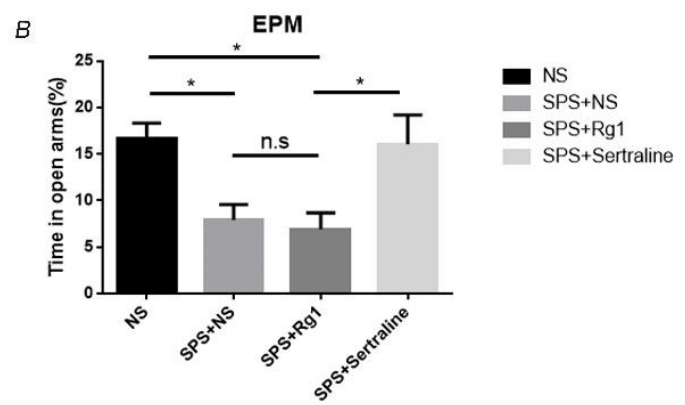

Figure 1. Comparison of experimental results of elevated plus maze(EPM) in mice in each group(A-B). (A) The number of times entered the open arms. The SPS+NS group and the SPS+Rg1 group were significantly reduced compared with the NS and SPS+Sertraline group and there was no significant difference between the SPS+NS group and the SPS+Rg1 group. (B) Time in open arms. The SPS+NS group and the SPS $+\operatorname{Rg} 1$ group were significantly reduced compared with the NS group, and the SPS+Rg1 group was significantly reduced compared with the SPS+Sertraline group . ${ }^{*} P<0.05, * * P<0.01$ and $* * * P<0.001$ mean significant difference, n.s. means not significant comparison, $\mathrm{n}=8$ mice for each group. All data are shown as means \pm SEM. 

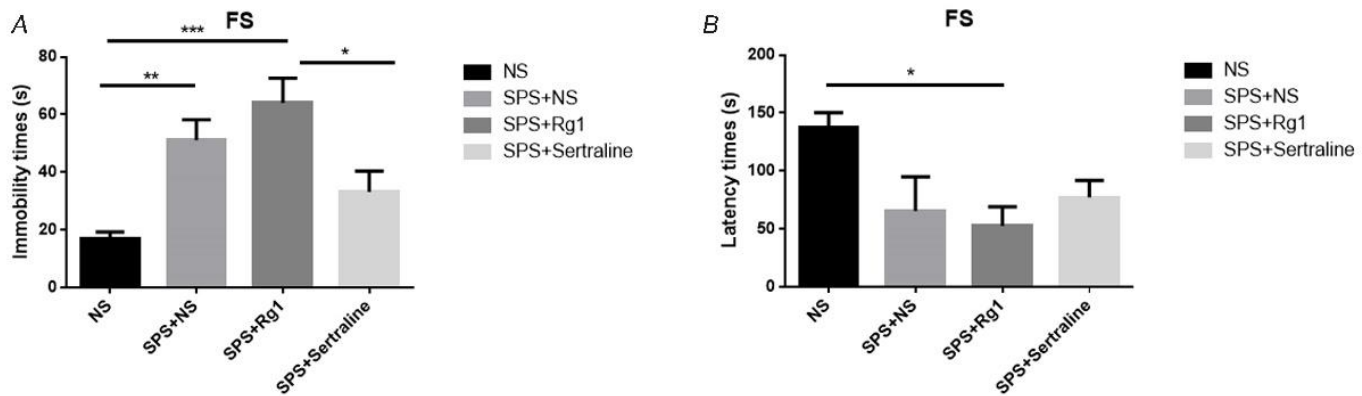

Figure 2. Comparison of the results of forced swimming(FS) among the groups (A-B). (A) Immobility time in forced swimming test. The immobility time of SPS+NS group and SPS+Rg1 group was significantly prolonged, and there was no significant difference between the SPS+NS group and the SPS+Rg1 group. Compared with the SPS+Sertraline group, the mice in the SPS+Rg1 group also had significantly longer inactivity time. (B) The latency time in forced swimming test. Compared with the NS group, the latency time of the SPS+Rg1 group was significantly reduced. $* P<0.05, * * P<0.01$ and $* * * P<0.001$ mean significant difference between groups, n.s. means not significant comparison, $\mathrm{n}=8$ mice for each group. All data are shown as means \pm SEM.

\section{Discussion}

Previous studies have shown that SSRIs have a preventive effect on PTSD and can alleviate PTSD related anxiety symptoms $s^{2,10,11}$ and reduce the immobilization time of forced swimming ${ }^{12}$, which is consistent with our study. This experiment also proved that sertraline can alleviate anxiety and fear symptoms. There are currently no studies on the prevention and treatment of PTSD with $\operatorname{Rg} 1$.Previous studies have shown that $\operatorname{Rg} 1$ can be used to treat acute and chronic depression and promote neurogenesis ${ }^{6}$, which has a protective effect on injured hippocampal neurons.But it's not contradiction with our study, the possible reasons are: (1) $\operatorname{Rg} 1$ improve activated brain derived neurotrophic factor (BDNF) in order to promote neurogenesis to treat depression, but PTSD and depression are two different diseases, etiology and pathogenesis are not completely consistent, chemical, biological changes in the body and brain are not completely consistent, such as better dexamethasone suppression test in PTSD, Rg1 can affect people with PTSD BDNF and after effects of BDNF on PTSD symptoms have reverse worth further discussing.(2) this experiment adopts the most appropriate dose according to the dose previously studied $^{6,13}$.It is speculated that the concentration and dosage of $\operatorname{Rg} 1$ may be insufficient, and the concentration of $\operatorname{Rg} 1$ can be increased for further study. In this study, the elevated cross maze experiment can correctly reflect the anxiety of mice ${ }^{14}$. After the stress was prolonged for a single time, the entry times and residence time of the open arm of the mice were significantly reduced, which could indicate that the exploratory behavior of the mice was reduced and the anxiety level was increased. After stress, sertraline was given as a preventive intervention, and the level of anxiety in mice was reduced. However, the symptoms of mice treated with ginsenoside $\mathrm{Rg} 1$ did not improve significantly, and there was no significant difference between the two groups. Forced swimming experiment can correctly reflect the fear of mice ${ }^{15}$.After the stress was prolonged for a single time, the inactivity time of the mice was prolonged and the incubation period was shortened, indicating that the fear level of the mice was increased. After stress, sertraline was given as a preventive intervention, and the fear level of the mice decreased. However, the symptoms of mice treated with ginsenoside $\operatorname{Rg} 1$ did not improve significantly.

This study shows that a single prolonged stress successfully simulates some symptoms of PTSD, such as anxiety and fear, with good reliability. Immediate intervention by SSRIs after stress has certain effect on the prevention of PTSD. In contrast, $\operatorname{Rg} 1$ had no significant effect on symptom improvement.

\section{Reference}

1. Eagle, A. L., Fitzpatrick, C. J. \& Perrine, S. A. Single prolonged stress impairs social and object novelty recognition in rats. Behavioural brain research 256, 591-597, doi:10.1016/j.bbr.2013.09.014 (2013).

2. Zhang, L. M. et al. Anxiolytic effects of flavonoids in animal models of posttraumatic stress disorder. Evidence-based complementary and alternative medicine : eCAM 2012, 623753, doi:10.1155/2012/623753 (2012).

3. Lane, R., Baldwin, D. \& Preskorn, S. The SSRIs: advantages, disadvantages and differences. Journal of psychopharmacology 9, 163-178, doi:10.1177/0269881195009002011 (1995).

4. Rudakewich, M., Ba, F. \& Benishin, C. G. Neurotrophic and neuroprotective actions of ginsenosides $\operatorname{Rb}(1)$ and $\operatorname{Rg}(1)$. Planta medica 67, 533-537, doi:10.1055/s-2001-16488 (2001).

5. Li, X. J. et al. Neuroprotective effects of tongluojiunao in neurons exposed to oxygen and glucose deprivation. Journal of ethnopharmacology 141, 927-933, doi:10.1016/j.jep.2012.03.042 (2012).

6. Jiang, B. et al. Antidepressant-like effects of ginsenoside Rg1 are due to activation of the BDNF signalling pathway and neurogenesis in the hippocampus. British journal of pharmacology 166, 1872-1887, doi:10.1111/j.1476-5381.2012.01902.x (2012).

7. Liberzon, I., Krstov, M. \& Young, E. A. Stress-restress: effects on ACTH and fast feedback. Psychoneuroendocrinology 22, 443-453 (1997).

8. Komada, M., Takao, K. \& Miyakawa, T. Elevated plus maze for mice. Journal of Visualized Experiments (2008). 
9. Thoeringer, $\mathrm{C}$. K. et al. The temporal dynamics of intrahippocampal corticosterone in response to stress-related stimuli with different emotional and physical load: an in vivo microdialysis study in C57BL/6 and DBA/2 inbred mice.

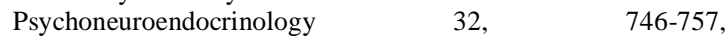
doi:10.1016/j.psyneuen.2007.05.005 (2007)

10. Qiu, Z. K. et al. Repeated administration of AC-5216, a ligand for the $18 \mathrm{kDa}$ translocator protein, improves behavioral deficits in a mouse model of post-traumatic stress disorder. Progress in neuro-psychopharmacology \& biological psychiatry 45, 40-46, doi:10.1016/j.pnpbp.2013.04.010 (2013)

11. Matar, M. A., Cohen, H., Kaplan, Z. \& Zohar, J. The effect of early poststressor intervention with sertraline on behavioral responses in an animal model of post-traumatic stress disorder Neuropsychopharmacology : official publication of the American College of Neuropsychopharmacology 31,
2610-2618, doi:10.1038/sj.npp.1301132 (2006).

12. Redrobe, J. P. \& Bourin, M. Dose-dependent influence of buspirone on the activities of selective serotonin reuptake inhibitors in the mouse forced swimming test. Psychopharmacology 138, 198-206 (1998).

13. $\mathrm{Xu}, \mathrm{S}$. F. et al. Improvement of ginsenoside $\operatorname{Rg} 1$ on hematopoietic function in cyclophosphamide-induced myelosuppression mice. European journal of pharmacology 695 7-12, doi:10.1016/j.ejphar.2012.07.050 (2012).

14. Lapiz-Bluhm, M. D. et al. Behavioural assays to model cognitive and affective dimensions of depression and anxiety in rats. Journal of neuroendocrinology 20, 1115-1137, doi:10.1111/j.1365-2826.2008.01772.x (2008).

15. Yamamoto, S. et al. Single prolonged stress: toward an animal model of posttraumatic stress disorder. Depression and anxiety 26, 1110-1117, doi:10.1002/da.20629 (2009). 